\title{
THE FITZHUGH-NAGUMO SYSTEM AS A MODEL OF HUMAN CARDIAC ACTION POTENTIALS
}

\author{
Amy D. Ngo *1 \\ ${ }^{1}$ Department of Mathematical Sciences, Rensselaer Polytechnic Institute, Troy, NY, USA
}

\begin{abstract}
In this paper, we aim to develop models of the action potentials of healthy human myocardial and pacemaker cells using the periodically forced Fitzhugh-Nagumo system. Pacemaker cells generate impulses which cause myocardial cells to contract, producing a heartbeat. Such impulses both cause and result from changes in membrane potential. Using eigenvalue stability analysis and the Hopf Bifurcation Theorem, we determined ranges of the two constants intrinsic to the system and the forcing amplitude for which the system has a unique, stable limit cycle. From simulations in MATLAB, we discovered that at forcing amplitudes near and greater than the maximum value which induces the limit cycle, the square wave and the cosine wave forced systems describe the behaviors of myocardial and pacemaker action potentials, respectively, with high fidelity.
\end{abstract}

\section{Introduction}

The living heart is an example of a relaxation oscillator, a term used to describe self-sustained nonlinear oscillators that behave non-sinusoidally. The heart spontaneously generates the electrical impulses that make it pulsate, so the heartbeat is self-sustained. The rate at which the heart generates and responds to these impulses fluctuates naturally, so the heartbeat, albeit repetitive, does not have a constant period. When the body experiences a physiological stimulus, such as stress, the heart responds by beating faster or slower to supply the body with adequate blood. This change in pace can occur abruptly or smoothly, depending on how the stimulus is applied. Once this stimulus is removed, the heart eventually returns to its normal pace.

These similarities have motivated many efforts to model electrical activity of the heart as relaxation oscillations. Our goal in this paper is to construct models of the action potentials of healthy human myocardial and pacemaker cells based on the Fitzhugh-Nagumo system of ordinary differential equations (ODEs), which describes neural impulses. This system is an appropriate model of cardiac action potentials because under certain conditions, it exhibits a unique, stable limit cycle [1. We will force the system with different periodic functions and identify the conditions under which the action potential predicted by the system captures the behavior of either pacemaker or myocardial action potential. These conditions are the type of forcing function and the values of the forcing amplitude.

To properly model the heart, an understanding of how it works is needed. In a normal heart, pacemaker cells in the sinoatrial node discharge the electrical impulses that make the heart beat, an action known as depolarization. Each heartbeat consists of two phases: systole and diastole. During diastole, the heart is relaxed as its chambers fill with blood. Pacemaker cells depolarize at the end

*ngoa2@rpi.edu,ngoxx133@umn.edu 
of diastole, when the chambers are full of blood. Myocardial cells conduct the impulse throughout the heart, causing it to contract. This contraction allows the heart to pump blood into arteries for transport throughout the body. Once the chambers are empty, the heart relaxes, and its chambers begin to refill with blood [2, 3, 4].

These electrical impulses arise from cationic concentration gradients that exist at the cardiac cell membrane. Ion-specific channels on the membrane regulate flow of cations into and out of the cells according to the membrane potential, which is defined as intracellular potential minus extracellular potential.

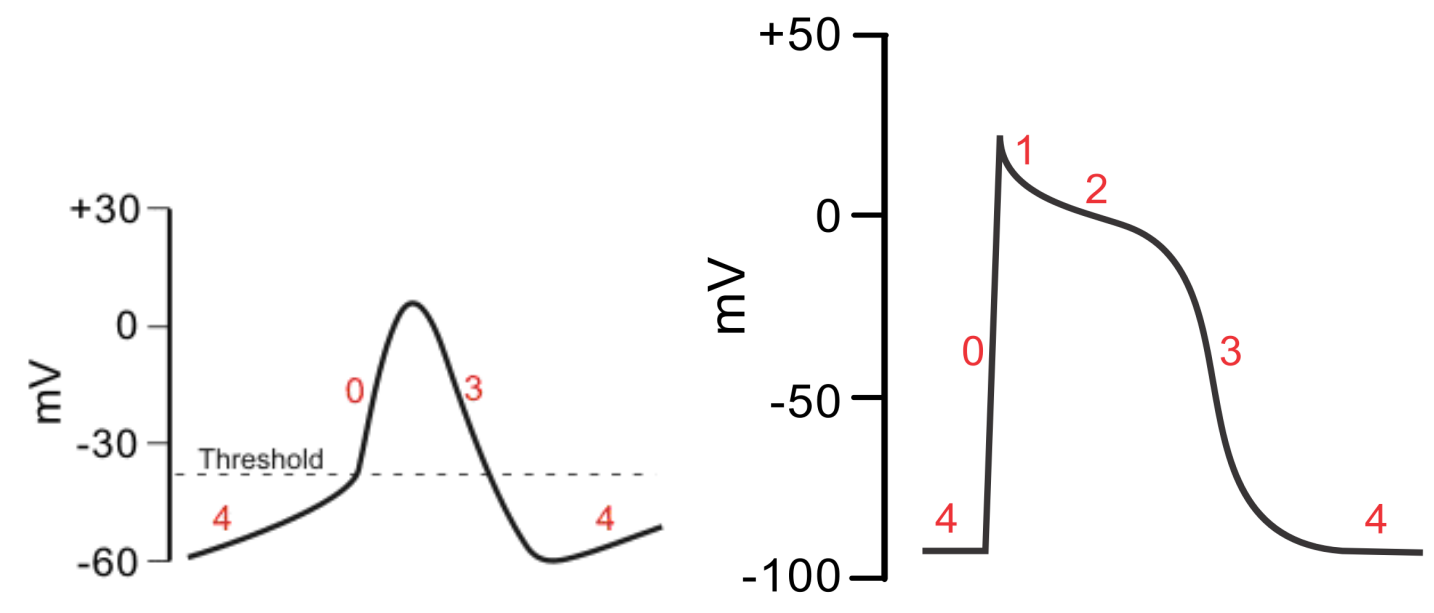

(a) A single pacemaker action potential [5]. (b) A single myocardial action potential [5].

Figure 1 above depicts a single pacemaker action potential (a) and a single myocardial action potential (b), both of which last around 300 msec. The most important regions of each action potential are numbered. During diastole (4), pacemaker cells have a membrane potential, known as resting potential in this phase, of -50 to $-60 \mathrm{mV}$. Sodium cations enter the cells until the membrane potential rises to the so-called threshold potential of about $-40 \mathrm{mV}$, at which point the cells depolarize spontaneously. Calcium cations then flow into the cells until the membrane potential peaks at $+10 \mathrm{mV}(0)$. When myocardial cells conduct the impulse (4), sodium cations enter the cells, and their membrane potential rises to about $+50 \mathrm{mV}(0)$, from the resting potential of -80 to $-90 \mathrm{mV}$. Calcium cations then enter the cells, triggering contraction of the heart, as some sodium cations exit the cells, causing membrane potential to initially drop (1), then plateau (2). As systole progresses, potassium cations exit both myocardial and pacemaker cells until their respective membrane potentials drop back to the resting potentials (3). This phase is known as repolarization. Once the impulse has traversed the heart, the heart relaxes and begins to refill with blood, and diastole starts anew [2, 4].

From Figure 1, we see that pacemaker action potential is characterized by sharp, wide peaks, while myocardial action potential behaves like square waves, each with a positive jump at the left end. These differences are due to the different nature of pacemaker and myocardial cells. The automaticity of pacemaker cells causes their membrane potential to rise and drop smoothly. In contrast, myocardial cells are not automatic, so their membrane potential does not change if impulses are absent and changes abruptly when an impulse becomes present.

The oscillations in these action potentials are non-sinusoidal, not unlike the oscillations observed in the current in a triode circuit. In the 1860s, Balthasar Van der Pol, when modeling the 
amplitude of this current, constructed one of the first systems of ODEs to exhibit non-sinusoidal oscillations, which is now known as the Van der Pol system [6, 7]:

$$
\begin{aligned}
& \frac{d x}{d t}=y-x^{3}+x, \\
& \frac{d y}{d t}=-x
\end{aligned}
$$

Here, $x$ is a scaled representation of the current, and $y$ quantifies forces that return $x$ to its initial state. The oscillations occurred without the need for forcing and consisted of jumps, corresponding to the rapid discharge of current by a capacitor in the circuit, separated by periods of gradual change during which the capacitor recharged. In forward time, they converged to a unique stable limit cycle. Van der Pol asserted that natural phenomena which were spontaneous and repetitive, such as the triode current and the "beating of the heart," but not as regular as the vibrations of the plucked strings of a musical instrument could all be modeled by relaxation oscillations [6].

In 1952, the Hodgkin-Huxley model of electrical impulses among cells was published. It was one of the most advanced such models of its time, having been constructed from data obtained from a squid giant axon. It has four state variables: membrane potential, sodium activation and inactivation, and potassium activation. (In)activation is a dimensionless quantity between 0 and 1 which describes how many (how few) cations are flowing through cells. The model accurately describes how membrane potential and cationic flow affect initiation and propagation of electrical impulses among different cells, including neurons and cardiac cells [8, 9. However, it is difficult to visualize or analyze qualitatively, being four-dimensional.

In 1960, Richard Fitzhugh reduced the system to two dimensions. Because the system had a unique fixed point and a unique stable limit cycle under certain conditions, Fitzhugh recognized that he could illustrate its most crucial characteristics in the two-dimensional phase plane with the Van der Pol system. He lumped membrane potential with sodium activation, which describe depolarization and repolarization, and sodium inactivation with potassium activation, which represent the inertia of membrane potential, then made several variable transformations to simplify the system to a Van der Pol-esque system. Modifications by Jin-Ichi Nagumo in 1962 transformed this system into the Fitzhugh-Nagumo model [9].

\section{The Fitzhugh-Nagumo Model}

The version of the Fitzhugh-Nagumo model studied in this paper is given by [7]

$$
\begin{gathered}
\frac{d x}{d t}=x^{\prime}=y+x-\frac{x^{3}}{3}+I(t), \\
\frac{d y}{d t}=y^{\prime}=-x+a-b y,
\end{gathered}
$$

where $x$ is the lumped membrane potential and sodium activation, $y$ is the lumped sodium inactivation and potassium activation, $t$ is time, $I(t)$ is electrical impulse as a function of time, and $a$ and $b$ are constants. Therefore, $x$ is analogous to membrane potential, while $y$ represents the cells' "accommodation and refractoriness," or their resistance to respond to stimuli [1]. The unit of $t$ is msec, while $x, y, a, b$, and $I$ are dimensionless. Here, $I$ is the magnitude of the impulse with sign, where the impulse is measured in $\mu \mathrm{A} / \mathrm{cm}^{2}$. To obtain physiologically sound results, Fitzhugh put the following constraints on $a$ and $b[7,8$ ]:

$$
0<\frac{3}{2}(1-a)<b<1 .
$$


Although the system has three possible parameters - $a, b$, and $I(t)$ - our main focus is on how $I(t)$ affects the behavior of the system. In this section, we present and prove theorems on the fixed point and solutions of the system as dependent on $I$, which we use to denote a constant value of $I(t)$.

\subsection{Fixed Point and Stability Analysis}

This section has two main results: the existence and uniqueness of the fixed point of the system, and the existence of a unique stable limit cycle within a certain range of values of $I$.

Theorem 1. Under the constraints stated in (3), the system always has a unique fixed point for a given value of $I$.

Proof. Suppose a value of $I$ is given. Setting (1) and (2) to zero yields, respectively, the x- and y-nullclines of the system:

$$
\begin{gathered}
y=\frac{x^{3}}{3}-x-I, \\
y=\frac{a-x}{b} .
\end{gathered}
$$

Since the fixed point is the point of intersection of the nullclines, we set (4) and (5) equal to each other, and rewrite the resulting expression as the equation below:

$$
f(x)=\frac{x^{3}}{3}+\left(\frac{1}{b}-1\right) x-\left(\frac{a}{b}+I\right) .
$$

We will use the continuity of $f$ and its derivative, $f^{\prime}$, to prove $f$ has a unique real root.

Basic theorems of real analysis state that every polynomial is continuous on $\mathbb{R}$. Furthermore, since the derivative of a polynomial is also a polynomial, it must also be continuous on $\mathbb{R}$. Thus, we conclude that $f$ and $f^{\prime}$ are continuous on $\mathbb{R}$.

Consequently, we can apply the Intermediate Value Theorem to $f(x)$. Consider the end behavior of $f(x)$. When $x \rightarrow+\infty, f(x) \rightarrow+\infty$, since both cubic and linear terms in $x$ have positive coefficients and $\frac{a}{b}$ is finite. For the same reason, $f(x) \rightarrow-\infty$ as $x \rightarrow-\infty$. Then by the Intermediate Value Theorem, there must exist at least one value $x_{0} \in(-\infty, \infty)$ such that $f\left(x_{0}\right)=0$. Thus, $\left(x_{0}, y_{0}\right)$, where $y_{0}$ may be found by substituting $x_{o}$ into either (4) or (5), is a fixed point of the system.

To prove that this fixed point is unique, we compute $f^{\prime}$ :

$$
f^{\prime}(x)=x^{2}+\frac{1}{b}-1
$$

Since $b<1, \frac{1}{b}>1$, so $f^{\prime}(x) \geq \frac{1}{b}-1>0$ for all $x \in \mathbb{R}$. Then $f$ increases monotonically and thus can cross the $\mathrm{x}$-axis at most once. Hence, the fixed point is unique.

We now state a theorem on the stability of the fixed point.

Theorem 2. For $I=0$ and all values of $a$ and $b$ satisfying (3), the fixed point of the system is asymptotically stable.

Proof. We use eigenvalue analysis to determine the stability of $\left(x_{0}, y_{0}\right)$. First, we compute the Jacobian matrix $J$ of the system at $\left(x_{0}, y_{0}\right)$ :

$$
\left.J\right|_{\left(x_{0}, y_{0}\right)}=\left.\left(\begin{array}{ll}
\frac{\partial x^{\prime}}{\partial x} & \frac{\partial x^{\prime}}{\partial y} \\
\frac{\partial y^{\prime}}{\partial x} & \frac{\partial y^{\prime}}{\partial y}
\end{array}\right)\right|_{\left(x_{0}, y_{0}\right)}=\left.\left(\begin{array}{cc}
1-x^{2} & 1 \\
-1 & -b
\end{array}\right)\right|_{\left(x_{0}, y_{0}\right)}=\left(\begin{array}{cc}
1-x_{0}^{2} & 1 \\
-1 & -b
\end{array}\right) .
$$


Next, we compute the characteristic polynomial of $J$ :

$$
\left|J_{\left(x_{0}, y_{0}\right)}-\lambda I\right|=\left|\begin{array}{cc}
\left(1-x_{0}^{2}\right)-\lambda & 1 \\
-1 & -b-\lambda
\end{array}\right|=\lambda^{2}+\left(b+x_{0}^{2}-1\right) \lambda+\left[b\left(x_{0}^{2}-1\right)+1\right] .
$$

The roots of this polynomial are the eigenvalues of $J$ and can be found using the quadratic formula:

$$
\lambda_{1,2}=\frac{1-\left(b+x_{0}^{2}\right) \pm \sqrt{\left(b+x_{0}^{2}-1\right)^{2}-4\left[b\left(x_{0}^{2}-1\right)+1\right]}}{2} .
$$

We claim that $\lambda_{1,2}$ are complex and that $\operatorname{Re}\left(\lambda_{1,2}\right)<0$.

To prove that $\operatorname{Re}\left(\lambda_{1,2}\right)<0$, we will show that the determinant of $J$, $\operatorname{det}(J)$, is positive and the trace of $J, \operatorname{tr}(J)$, is negative. From (8) and the fact that $b<1$, we see that $\operatorname{det}(J)$ is given by

$$
\operatorname{det}(J)=1-b\left(1-x_{0}^{2}\right)=1+x_{0}^{2}-b>1-b>0,
$$

as desired. Now from (10), we see that $\operatorname{tr}(J)$ is given by

$$
\operatorname{tr}(J)=1-\left(b+x_{0}^{2}\right) .
$$

To prove $1-\left(b+x_{0}^{2}\right)<0$, we suppose that $0 \leq x_{0} \leq \sqrt{1-b}$. Substituting $x_{0}$ into (6) yields

$$
\begin{aligned}
f\left(x_{0}\right) \leq \frac{(1-b)^{3 / 2}}{3}+\left(\frac{1}{b}-1\right) \sqrt{1-b}-\frac{a}{b} & =\frac{\sqrt{1-b}}{b}\left(\frac{b}{3}-\frac{b^{2}}{3}+1-b\right)-\frac{a}{b} \\
& <\frac{1}{b}\left(1-\frac{2 b}{3}-\frac{b^{2}}{3}\right) \quad \text { since } b<1 \\
& <\frac{1}{b}\left(a-\frac{b^{2}}{3}\right)-\frac{a}{b} \quad \text { since } 1-\frac{2}{3} b<a \\
& =-\frac{b}{3}<0 .
\end{aligned}
$$

Thus, $f\left(x_{0}\right)<0$, which is a contradiction, as $x_{0}$ is supposed to satisfy $f\left(x_{0}\right)=0$. Then since $x_{0} \not \leq \sqrt{1-b}$, we must have $x_{o}>\sqrt{1-b}$, and so $\operatorname{tr}(J)<0$.

Now, to prove that $\lambda_{1,2}$ are complex, let's first evaluate the discriminant of (9):

$$
\begin{aligned}
\left(x_{0}^{2}+b-1\right)^{2}-4\left[b\left(x_{0}^{2}-1\right)+1\right] & =x_{0}^{4}+2(b-1) x_{0}^{2}+(b-1)^{2}-4 b\left(x_{0}^{2}-1\right)-4 \\
& =x_{0}^{4}-2(b+1) x_{0}^{2}+b^{2}+2 b-3=\left[x_{0}^{2}-(b-1)\right]\left[x_{0}^{2}-(b+3)\right] .
\end{aligned}
$$

We know $x_{0}^{2}>1-b$, so to prove the discriminant is negative, we need to prove $x_{0}^{2}<b+3$. To this end, we suppose that $x_{0} \geq \sqrt{b+3}$ and substitute $x_{0}$ into (6):

$$
\begin{aligned}
f\left(x_{0}\right) \geq \sqrt{b+3}\left(\frac{b+3}{b}+\frac{1}{b}-1\right)-\frac{a}{b} & =\frac{\sqrt{b+3}}{b}\left(\frac{b^{2}}{3}+b-1-b\right)-\frac{a}{b} \\
& >\frac{\sqrt{3}}{b}\left(\frac{b^{2}}{3}+1\right)-\frac{a}{b}>\frac{1-a}{b}>0 \quad \text { since } a<1 .
\end{aligned}
$$

Thus, $f\left(x_{0}\right)>0$, which is a contradiction, as $x_{0}$ is supposed to satisfy $f\left(x_{0}\right)=0$. Then since $x_{0} \nsucceq \sqrt{b+3}$, we must have $x_{0}<\sqrt{b+3}$, so that the discriminant is negative and $\lambda_{1,2}$ are complex.

Therefore, $\left(x_{0}, y_{0}\right)$ is hyperbolic and asymptotically stable.

We now provide the values of $I$ for which the fixed point is unstable, as dependent on $a$ and $b$. 
Proposition 1. For values of $a$ and $b$ satisfying (3), the fixed point of the system is unstable if the value of I satisfies

$$
-\frac{\sqrt{1-b}}{b}\left[1-\frac{b}{3}(b+2)\right]-\frac{a}{b}<I<\frac{\sqrt{1-b}}{b}\left[1-\frac{b}{3}(b+2)\right]-\frac{a}{b} .
$$

Proof. From (10), we see the fixed point is unstable if $x_{0}$ satisfies $-\sqrt{1-b}<x_{0}<\sqrt{1-b}$, where $\operatorname{Re}\left(\lambda_{1,2}\right)>0$. From $(7)$, we know that $f(x)$ is a monotonically increasing function, so since $f\left(x_{0}\right)=0$, the following inequalities hold:

$$
\begin{aligned}
& f(-\sqrt{1-b})=-\frac{\sqrt{1-b}}{b}\left[1-\frac{b}{3}(b+2)\right]-\left(\frac{a}{b}+I\right)<0 \\
& f(\sqrt{1-b})=\frac{\sqrt{1-b}}{b}\left[1-\frac{b}{3}(b+2)\right]-\left(\frac{a}{b}+I\right)>0 .
\end{aligned}
$$

We easily obtain (11) by rearranging these inequalities.

These results lead us to the following theorem.

Theorem 3. When the fixed point of the system is unstable, the system has a unique $\omega$-limit cycle.

Proof. For this proof, we use the Hopf Bifurcation Theorem (HBT) [11]. A Hopf bifurcation occurs at $x_{0}= \pm \sqrt{1-b}$, where $\lambda_{1,2}$ are imaginary, as (10) and the proof of Theorem 2 indicate. The stability of the fixed point changes from stable to unstable at $x_{0}=-\sqrt{1-b}$. We claim that a supercritical bifurcation occurs at the fixed point with this value of $x_{0}$, and we let $\lambda_{1,2}$ denote the eigenvalues evaluated at this fixed point.

The first condition of HBT is that $\lambda_{1,2}$ must be non-hyperbolic. As we mentioned, $\operatorname{Re}\left(\lambda_{1,2}\right)=0$, so $\lambda_{1,2}$ are indeed non-hyperbolic.

To determine whether $\lambda_{1,2}$ satisfy the transversality condition of HBT, we need to check if

$$
\tau=\left.\frac{d \operatorname{Re}\left(\lambda_{1,2}\right)}{d I}\right|_{I=I_{0}}=\left.\frac{d}{d I}\left(\frac{1-b-x_{0}^{2}}{2}\right)\right|_{I=I_{0}}=-\left.x_{0} \frac{d x_{0}}{d I}\right|_{I=I_{0}} \neq 0,
$$

where $x_{0}=-\sqrt{1-b}$ if $I=I_{0}$. To compute $\frac{d x_{0}}{d I}$, we evaluate (6) at $x_{0}$ and implicitly differentiate $x_{0}$ with respect to $I$ :

$$
\begin{aligned}
f\left(x_{0}\right)=\frac{x_{0}^{3}}{3}+\left(\frac{1}{b}-1\right) x_{0}-\left(\frac{a}{b}+I\right)=0 \Rightarrow & x_{0}^{2} \frac{d x_{0}}{d I}+\left(\frac{1}{b}-1\right) \frac{d x_{0}}{d I}-1=0 \\
& \Rightarrow \quad \frac{d x_{0}}{d I}=\frac{1}{x_{0}^{2}+\frac{1}{b}-1} .
\end{aligned}
$$

Therefore, we find that

$$
\tau=\sqrt{1-b} \frac{1}{1-b+\frac{1}{b}-1}=\frac{\sqrt{1-b}}{\frac{1}{b}-b}>\sqrt{1-b}>0,
$$

which indicates $\lambda_{1,2}$ satisfies the transversality condition.

The last condition of HBT is genericity, which is defined as

$$
g=\frac{x_{x x x}^{\prime}+x_{x y y}^{\prime}+y_{x x y}^{\prime}+y_{y y y}^{\prime}}{16}+\left.\frac{x_{x y}^{\prime}\left(x_{x x}^{\prime}+x_{y y}^{\prime}\right)-y_{x y}^{\prime}\left(y_{x x}^{\prime}+y_{y y}^{\prime}\right)-x_{x x}^{\prime} y_{x x}^{\prime}+x_{y y}^{\prime} y_{y y}^{\prime}}{16 \operatorname{Im}\left(\lambda_{1,2}\right)}\right|_{I=I_{0}} \neq 0 .
$$


The only nonzero partial derivatives of $x^{\prime}$ and $y^{\prime}$ are

$$
x_{x}^{\prime}=1-3 x^{2}, \quad x_{x x}^{\prime}=-6 x, \quad x_{x x x}^{\prime}=-6, \quad x_{y}^{\prime}=1 ; \quad y_{x}^{\prime}=-1 ; \quad y_{y}^{\prime}=-b .
$$

Then $g=-6 \neq 0$, and so the system satisfies the genericity condition.

Since $\tau g=-6 \sqrt{1-b}<0$ and the fixed point is unstable, the Hopf bifurcation we mentioned is supercritical, and a unique stable limit cycle exists when $-\sqrt{1-b}<x_{0}<\sqrt{1-b}$.

\section{Numerical Analysis}

We use conditions (2) and (11) from the previous section to choose values of $a, b$, and $I$ for simulations of the system. Following Fitzhugh and Nagumo's definition, $I$ is negative when the system depolarizes and positive when the system conducts an impulse 8 .

To generate phase portraits of the system, we use the fourth-order Runge-Kutta (RK4) method with a forward time step of 0.01 msec. We restrict the initial conditions in $\mathrm{x}$ and $\mathrm{y}$ to the ranges $[-2,2]$ and $[-1,1]$, respectively, as this region suffices to contain Fitzhugh's projections of the entire Hodgkin-Huxley model state space onto the two-dimensional phase plane [8, 10].

We study four cases of the system: $I=0 ; I$ is constant and induces a limit cycle; $I$ is a square wave; $I$ is a cosine wave. Our goal in studying every case is to identify a suitable model for either action potential. The first two cases provide insight on the possible attractors - fixed point and limit cycle - of the system. Using this insight, we then study the square wave forced system as a model for myocardial action potential and the cosine wave forced system as a model for pacemaker action potential. From the following results, we conclude that the square wave and the cosine wave forced systems are more consistent models of the action potentials.

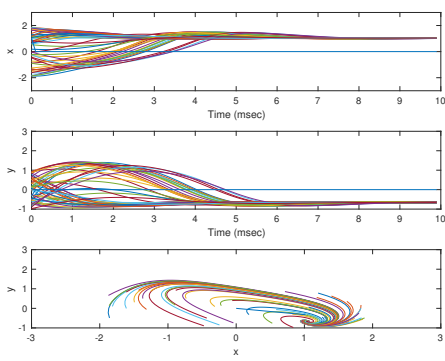

(a) $a=0.4, b=0.975$

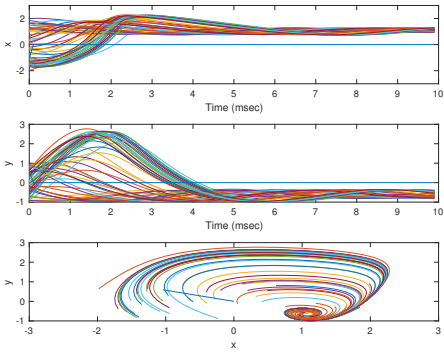

(b) $a=0.9, b=0.25$

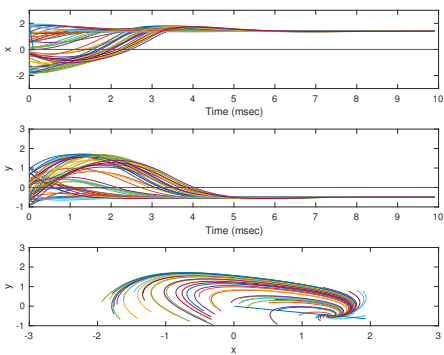

(d) $a=0.9, b=0.975$

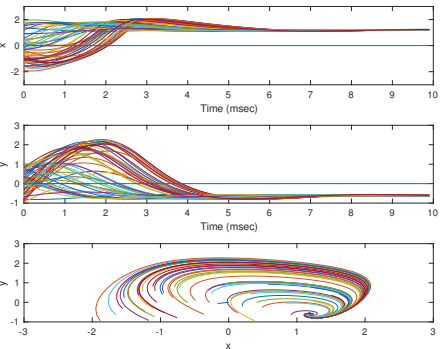

(c) $a=0.9, b=0.5$

Figure 2: Phase portraits of the free system at different values of $a$ and $b$.

First, we study the system when $I=0$. Figure 2 above shows that the solutions of this system converge to the stable fixed point in forward time, as expected from Theorem 2. It also shows that 
increasing $b$ with $a$ fixed results in monotonic decrease in convergence time of the solutions, which is defined as time elapsed before the solutions appear to have converged to the fixed point. In contrast, changing $a$ with $b$ fixed has little effect on the solutions. For instance, convergence time decreases from $\sim 15 \mathrm{msec}$ to $\sim 6 \mathrm{msec}$ when $b$ increases from 0.25 to 0.975 at $a=0.9$, whereas convergence time changes from $\sim 8 \mathrm{msec}$ to $\sim 6 \mathrm{msec}$ when $a$ increases from 0.4 to 0.9 at $b=0.975$. Equation 2 explains why: $y^{\prime}$ is proportional to $-b y$ but has only a constant relationship with $a$. Increasing $b$ thus decreases the refractoriness of the system, making it more inclined to diverge from its initial state to the fixed point.

Since the solutions of the system converge to a single point, we conclude that this system is not representative of the entire behavior of cardiac action potentials. Rather, it is at most representative only of myocardial cells or pacemaker cells undergoing repolarization.

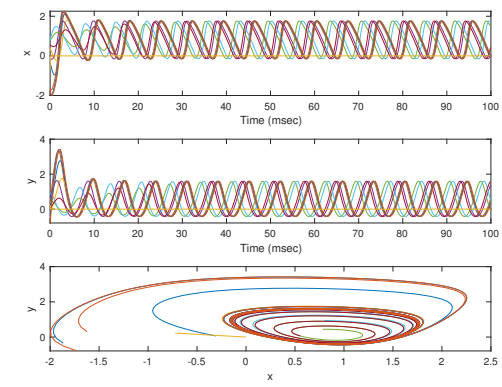

(a) $I=-0.75$

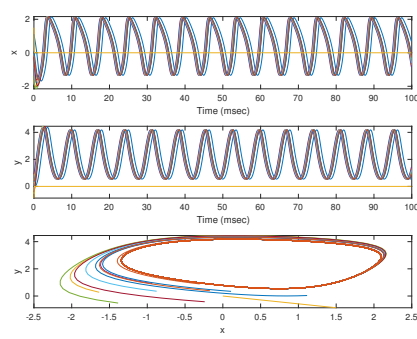

(b) $I=-2$
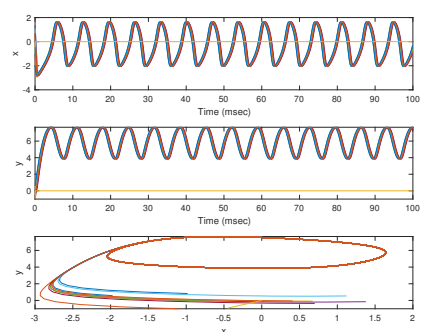

(c) $I=-6$
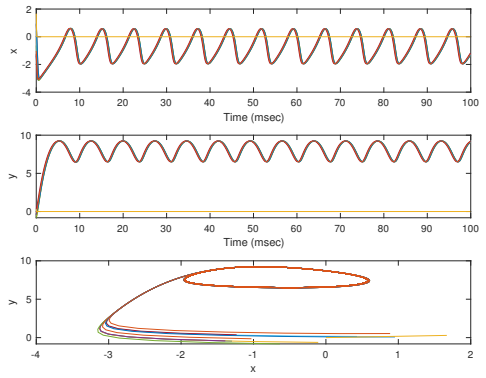

(d) $I=-8$

Figure 3: Phase portraits of the forced system when it has a limit cycle, $a=0.9, \& b=0.2$.

Next, we study the system when $I$ is constant and induces the limit cycle. Figure 3 above shows that solutions of the system converge asymptotically to the limit cycle in forward time, as expected from Theorem 3. We consider the system only for $a=0.9$ and $b=0.2$ because the range of $I(-8.32<I<-0.08)$ as given by $(11)$ is widest for these values and thus allows the most insightful analysis of how $I$ affects solutions of the system. As $I$ decreases, the amplitude of the oscillations initially increases, then decreases. This change is due to the fact that as $I$ approaches the lower bounds of the range, the fixed point approaches a supercritical Hopf bifurcation where its stability would change from unstable to stable, and the limit cycle would cease to exist.

The sharp peaks in $x$ appear similar to the peaks in pacemaker action potential. However, $x$ lacks time lags between successive peaks, as $x$ rises almost immediately after hitting its troughs. Such time lags are a crucial element of pacemaker action potential, as membrane potential changes much more gradually when pacemaker cells are in the refractory period than when they undergo 
depolarization. Hence, we conclude that $x$ is more representative of the action potential of coupled neuronal cells that generate impulses incessantly [10]. This conclusion is mathematically consistent: since $I$ is always negative, the system is always generating impulses.

To modify the system so that it models myocardial action potential, we observe that the action potential is characterized by jumps, both positive and negative, and plateaus. We observe from the previous systems that jumps are a feature of the forced system and plateaus occur only in the free system, where the fixed point is stable. Therefore, we hypothesize that the system can replicate the behavior of the action potential if it is forced in such a way that its fixed point is stable.

To this end, we study the system when $I(t)$ is a square wave with amplitude $A$ and period of $300 \mathrm{msec}$, which is the duration of a single cardiac action potential, for a wide range of values of $A$. Specifically, $I(t)$ is given by

$$
I(t)=\left\{\begin{array}{lr}
-A, & 0 \leq t<150 \\
A, & 150 \leq t<300
\end{array}\right.
$$

In the RK4 algorithm used to simulate the system, we express $I(t)$ as a Fourier sine series to prevent inaccuracies that could arise from the jump discontinuities in $I(t)$.
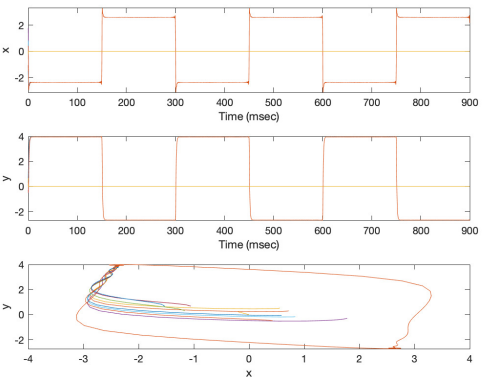

(a) $a=0.6, b=0.75, A=-6$
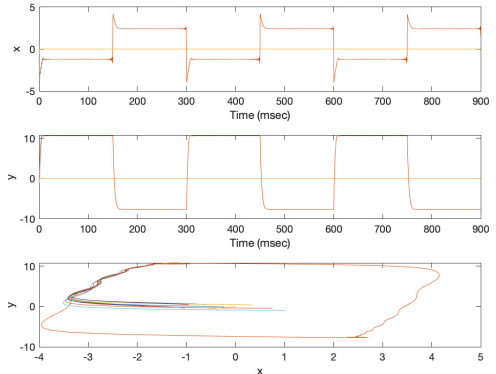

(c) $a=0.9, b=0.2, A=-10$
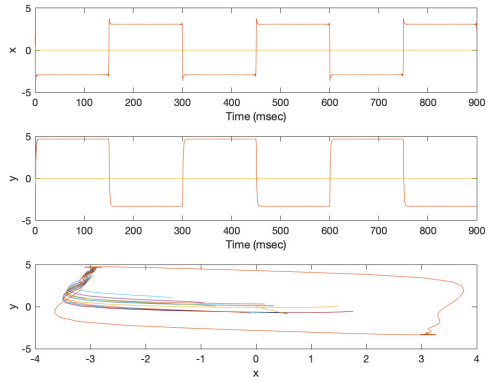

(b) $a=0.6, b=0.75, A=-10$
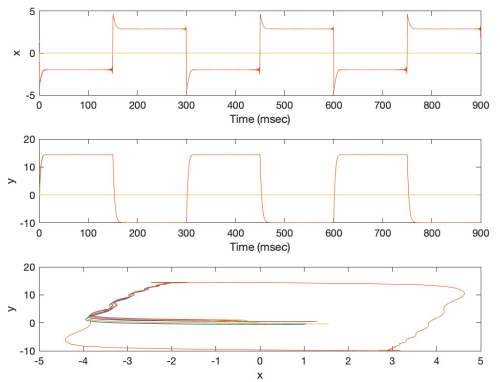

(d) $a=0.9, b=0.2, A=-15$

Figure 4: Phase portraits of the system when $I(t)$ is a square wave of amplitude $A$.

Figure 4 above confirms our hypothesis, as it shows that $x$ exhibits square wave-like behavior when $A$ is below the lower bound of the range given by (11). The fixed point is therefore stable both when $I(t)=A$ and when $I(t)=-A$. The large jump in $x$ at each half-period results from the change in magnitude of $I(t)$ of size $2 A$.

Figure 4 also shows that $x$ behaves similar to myocardial action potential. Each positive jump in $x$ is followed by a smaller negative jump and a lengthy plateau, corresponding to when myocardial 
cells conduct an impulse and cations then flow in and out of them as they contract. Afterward, $x$ experiences a large negative jump before plateauing at a negative value, corresponding to when myocardial cells repolarize, enter a refractory period, and then enter a resting period. The most notable difference between $x$ and myocardial action potential is that every negative jump in $x$ is followed by a smaller positive jump. The large, sudden change in $I(t)$ that precedes the negative jump causes $x$ to overshoot the fixed point before converging to it.

Now, to modify the system so that it models pacemaker action potential, we observe that the action potential behave similar to cosine waves. Recall that we deemed the constant forced system was not a suitable model of the action potential because $x$ rises immediately from its troughs. Additionally, Figure 4 indicates that the system responds almost, if not, instantaneously to changes in $I$. Therefore, we test whether the system can replicate the behavior of the action potential if its forcing function changes continually in time, like a cosine wave.

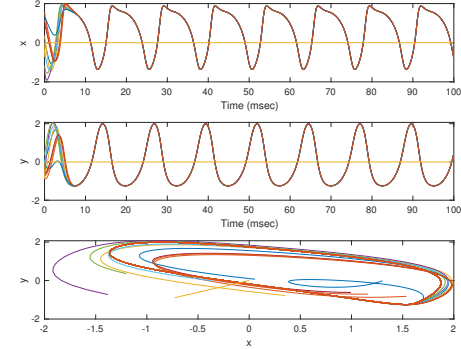

(a) $a=0.6, b=0.75, A=-0.9$
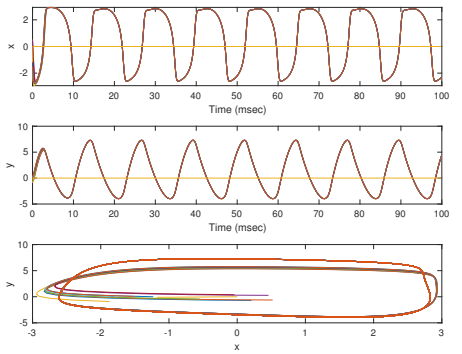

(d) $a=0.9, b=0.2, A=-6$
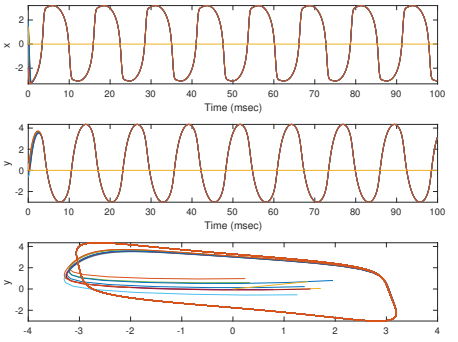

(b) $a=0.6, b=0.75, A=-10$
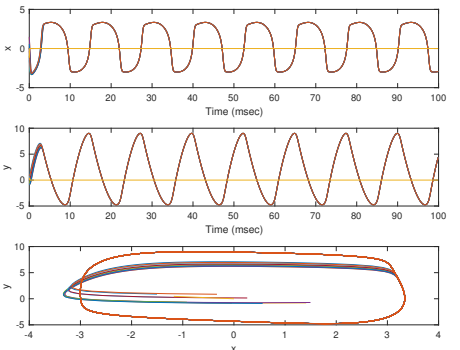

(e) $a=0.9, b=0.2, A=-10$

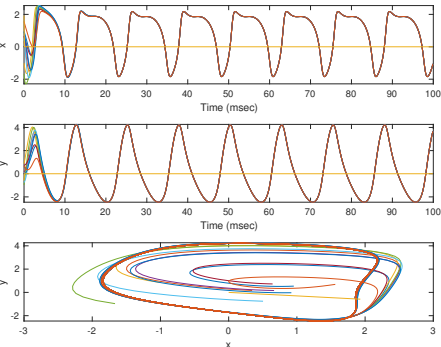

(c) $a=0.9, b=0.2, A=-2$
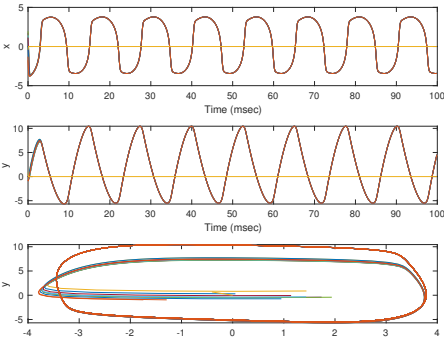

(f) $a=0.9, b=0.2, A=-15$

Figure 5: Phase portraits of the system where $I(t)=-A \cos w t$ and $w=0.5$.

To this end, we study the system when $I(t)=-A \cos w t$. We set $w=0.5$ so that not too many oscillations occur in a given timespan, which would complicate visual analysis of the phase portraits of the system. We could not choose $w$ so that the period of $I(t)$ is $300 \mathrm{msec}$, as doing so would distort the phase portraits. Figure 5 above shows that solutions of the system are phase-locked with $I(t)$, evident by their common period of roughly $13 \mathrm{msec}$. For small magnitudes of $A, x$ changes more gradually at its crests than at its troughs. In contrast, for larger magnitudes of $A, x$ changes at roughly the same rate at its crests and troughs. Relating the behavior of $x$ to the fixed point of the system is difficult, if not impossible. Since $I(t)$ changes continually in time, the fixed point, and likely its stability, is different from one value of $t$ to the next.

Figure 5 also shows that for large magnitudes of $A, x$ behaves similar to pacemaker action potential. Following a period of gradual increase from its trough, $x$ jumps to near its crest value, corresponding to the period where the action potential rises from the resting potential to the peak potential. After cresting, $x$ jumps back to near its trough value, corresponding to the period where 
pacemaker cells depolarize, then repolarize. However, there are some key differences between $x$ and the action potential. The action potential rises from its trough in a ramp-like manner, while $x$ does so more gradually in a wave-like manner. Likewise, after cresting, the action potential decreases almost immediately, while $x$ decreases more gradually, again in a wave-like manner. These differences stem from the fact that $x$ behaves like its cosine forcing function.

\section{Discussion}

In this work, we have used the periodically forced Fitzhugh-Nagumo system to construct models of healthy human cardiac action potentials.

Our most important result is that at high forcing amplitudes, the square wave and the cosine wave forced systems reproduce the waveforms of myocardial and pacemaker action potentials, respectively, with high fidelity. The square wave forced system captures how myocardial membrane potential jumps positively when the cells conduct an impulse, then decreases and plateaus as the cells contract, then jumps negatively back to the resting potential and plateaus there as the cells repolarize and return to the resting period. The cosine wave forced system captures most of the spiky behavior of pacemaker action potential and the more gradual behavior that occurs between the spikes. The membrane potential of pacemaker cells rises slowly from the resting potential to the threshold potential, then spikes to its crest, where depolarization occurs, before dropping rapidly back to the resting potential as the cells undergo repolarization.

As we explained in the previous section, each model has some discrepancies with the action potential it describes. Myocardial action potential does not increase just before dropping to its resting potential, whereas $x$ in the square wave forced system exhibits a small positive jump before plateauing at a negative value. Pacemaker action potential changes more rapidly at its crests and troughs than $x$ in the cosine wave forced system does. These discrepancies are the result of the system's response to the forcing function in each model. We will now describe the inherent weaknesses of each model.

In the square wave forced system, $I(t)$ is nonzero over the regions where $x$ plateaus. When $x$ is at its positive plateau value, $I(t)$ is positive, and likewise, when $x$ is at its negative plateau value, $I(t)$ is negative. By definition of the model, $I(t)$ is positive when cations flow into cells and negative when cations flow out of cells. However, when myocardial action potential is at its positive plateau value, cations flow both into and out of the cells in such a way that membrane potential is approximately constant. Therefore, $I(t)$ should have a net value of zero in this case. The same is true for when the action potential plateaus at the resting potential, as cations do not flow into or out of the cells when the cells are at rest.

In the cosine wave forced system, $x$ is synchronous with $I$, so that the system is always under forcing, except at the midpoint of each period where $I=0$. While this ubiquity of forcing appears to contradict the automaticity of pacemaker cells, which can depolarize even in the absence of impulses, the exact mechanism behind this automaticity remains unknown. Recent research suggests that this automaticity is the result of so-called clocks in the body which regulate the activity of the membrane ion channels of pacemaker cells and thus cationic flow into and out of the cells. These clocks are cyclical, not unlike the forcing function in our model [12. If such clocks exist, then the system would be biologically consistent with pacemaker action potential if we reinterpret the forcing as the regulatory activity of these clocks, rather than, for instance, impulses generated by different cells, as the model's definition of $I(t)$ implies.

The weaknesses we have described arise from the fact that $x$ and $y$ are not exact quantitative representations of membrane potential and cationic flow in cells. The main purpose of the 
construction of the Fitzhugh-Nagumo system was so that the system exhibits relaxation oscillations and a unique limit cycle which behave like those of the Hodgkin-Huxley system [8, 10. Hence, it is unlikely that the Fitzhugh-Nagumo system can explain every quantitative or qualitative intricacy of cellular electrical activity.

Despite these weaknesses, we conclude that the models we have constructed are useful to future studies of cardiac action potentials, as each model is in close qualitative agreement with the action potential it describes. Given that the models are easy to visualize, analyze mathematically, and modify, they could be used as a framework for developing more quantitatively and biologically accurate models of cardiac action potentials.

\section{References}

[1] Fitzhugh, R. Impulses and Physiological States in Theoretical Models of Nerve Membrane. Biophysical Journal, 1 (6): 445-466, 1961.

[2] Homoud, M.K. Introduction to Electrocardiography. Tufts-New England Medical Center, 2008.

[3] Silverman, M.E., Grove, D., Upshaw Jr., C.B. Why Does the Heart Beat? The Discovery of the Electrical System of the Heart. Circulation, 113: 2775-2781, 2006.

[4] Pinnell, J., Turner, S., Howell, S. Cardiac muscle physiology. Continuing Education in Anaesthesia, Critical Care, \& Pain, 7 (3): 85-88, 2007.

[5] Klabunde, R.E. Cardiovascular Physiology Concepts. Philadelphia, PA: Lippincott Williams \& Wilkins, 2nd Ed., 2012, 9-40. https://www.cvphysiology.com/Arrhythmias/A004. https:// www.cvphysiology.com/Arrhythmias/A006.

[6] Ginoux, J.M., Letellier, C. Van der Pol and the history of relaxation oscillations: toward the emergence of a concept. Chaos, American Institute of Physics, 22: 23-120, 2012.

[7] Hirsch, M.W., Smale, S., Devaney, R.L. Differential Equations, Dynamical Systems, and an Introduction to Chaos. Waltham, MA: Academic Press, 4th Ed., 2013, 272-273.

[8] Edelstein-Keshet, L. Mathematical Models in Biology. New York, NY: McGraw-Hill, 1987, 311-341.

[9] Nagumo, J., Arimoto, S., Yoshizawa, S. An Active Pulse Transmission Line Simulating Nerve Axon. Proceedings of the IRE, 50 (10): 2061-2070, 1962.

[10] Murray, J.D. Mathematical Biology: I. An Introduction. New York, NY: Springer, 3rd Ed., 2000, 239-244.

[11] Heijden, G.V.D. Hopf bifurcation. Lecture notes. https://www.ucl.ac.uk/ ucesgvd/hopf.pdf.

[12] Joung, B., Ogawa, M., Lin, S.F., Chen, P.S. The Calcium and Voltage Clocks in Sinoatrial Node Automaticity. Korean Circulation Journal, 39 (6), 217-222, 2009. 